\title{
Resolviendo una discusión y un misterio sobre la trucha marrón, Salmo trutta (Linnaeus, 1758) en isla Navarino, Magallanes, Chile
}

\author{
Solving a discussion and a mystery about brown trout, \\ Salmo trutta (Linnaeus, 1758) in Navarino Island, Magallanes, Chile
}

Alan Maldonado-Márquez ${ }^{1,3,4}$, Javier Rendoll-Cárcamo ${ }^{1,2,3}$ \& Tamara Contador Tal,2,4 $^{1,3}$

La introducción de especies exóticas de peces, principalmente de salmónidos como Oncorhynchus mykiss (Walbaum, 1792) y Salmo trutta (Linnaeus, 1758) (Basulto, 2003), ha sido promovida en Chile desde inicios del siglo XX. Las poblaciones más abundantes de estas especies se registran a partir de la latitud $36^{\circ} \mathrm{S}$ hacia el sur (Soto et al. 2006). Su introducción se justificó debido a la baja riqueza de especies presentes en las cuencas continentales (Basulto, 2003), valor deportivo-alimenticio (Campos, 1970), nichos "vacíos o insaturados" que brindaban condiciones ideales para los salmónidos (Campos, 1970; Soto et al. 2006).

La introducción de $S$. trutta en la ecorregión subantártica de Magallanes se origina a partir del año 1927 (Vila et al. 1999) y al menos durante 12 años consecutivos se sembró una cantidad de 1,7 millones de ovas de esta especie y de otras como O. mykiss, Salvelinus fontinalis (Mitchill, 1814) y Salmo salar (Linnaeus, 1758) (Traba \& Ríos, 1985). Los antecedentes históricos y bibliográficos sobre la introducción de salmónidos en esta ecorregión son escasos, por lo que se dificulta realizar un catastro con información sobre los años de introducción de las especies y cuencas específicas, presentando un reto para comprender los reales efectos de estas especies invasoras sobre la fauna nativa de peces y macroinvertebrados.

La isla Navarino se encuentra al sur de Tierra del Fuego, inmersa en la ecorregión subantártica de Magallanes y en la Reserva de la Biosfera Cabo de Hornos (RBCH) (54-56 $\left.{ }^{\circ} \mathrm{S}\right)$.
La reserva tiene como objetivo ser un territorio destinado a la protección de los ecosistemas debido a las presiones del cambio medioambiental global (Rozzi et al. 2006). Sin embargo, su condición geográfica remota no está exenta de las amenazas del cambio medioambiental global, como el interés de la industria salmonicultora de posicionarse en el canal Beagle, ausencias de barreras fito y zoosanitarias, construcción de nuevas rutas para conectividad terrestre (ruta Vicuña-Yendegaia) y un nuevo muelle en isla Navarino para conexión marítima (Yendegaia-Puerto Williams), permitiendo el movimiento de especies potencialmente invasoras. Recientemente algunas de estas amenazas han sido documentadas, entre las cuales se encuentra la introducción de salmónidos en la RBCH (Maldonado-Márquez et al. 2020).

Los últimos estudios que hacen referencia a poblaciones de ictiofauna continental en isla Navarino corresponden a Anderson et al.

1 Laboratorio Wankara de Ecosistemas Dulceacuícolas Subantárticos y Antárticos, Programa de Conservación Biocultural Subantártica, Parque Etnobotánico Omora, Universidad de Magallanes, Puerto Williams, Chile.

$\Sigma$ alan.maldonado@umag.cl

2 Programa de Doctorado en Ciencias Antárticas y Subantárticas, Universidad de Magallanes, Punta Arenas, Chile.

3 Instituto de Ecología y Biodiversidad, Estación de Campo Parque Omora, Puerto Williams, Chile.

4 Núcleo Milenio de Salmónidos Invasores (INVASAL), Concepción, Chile. 


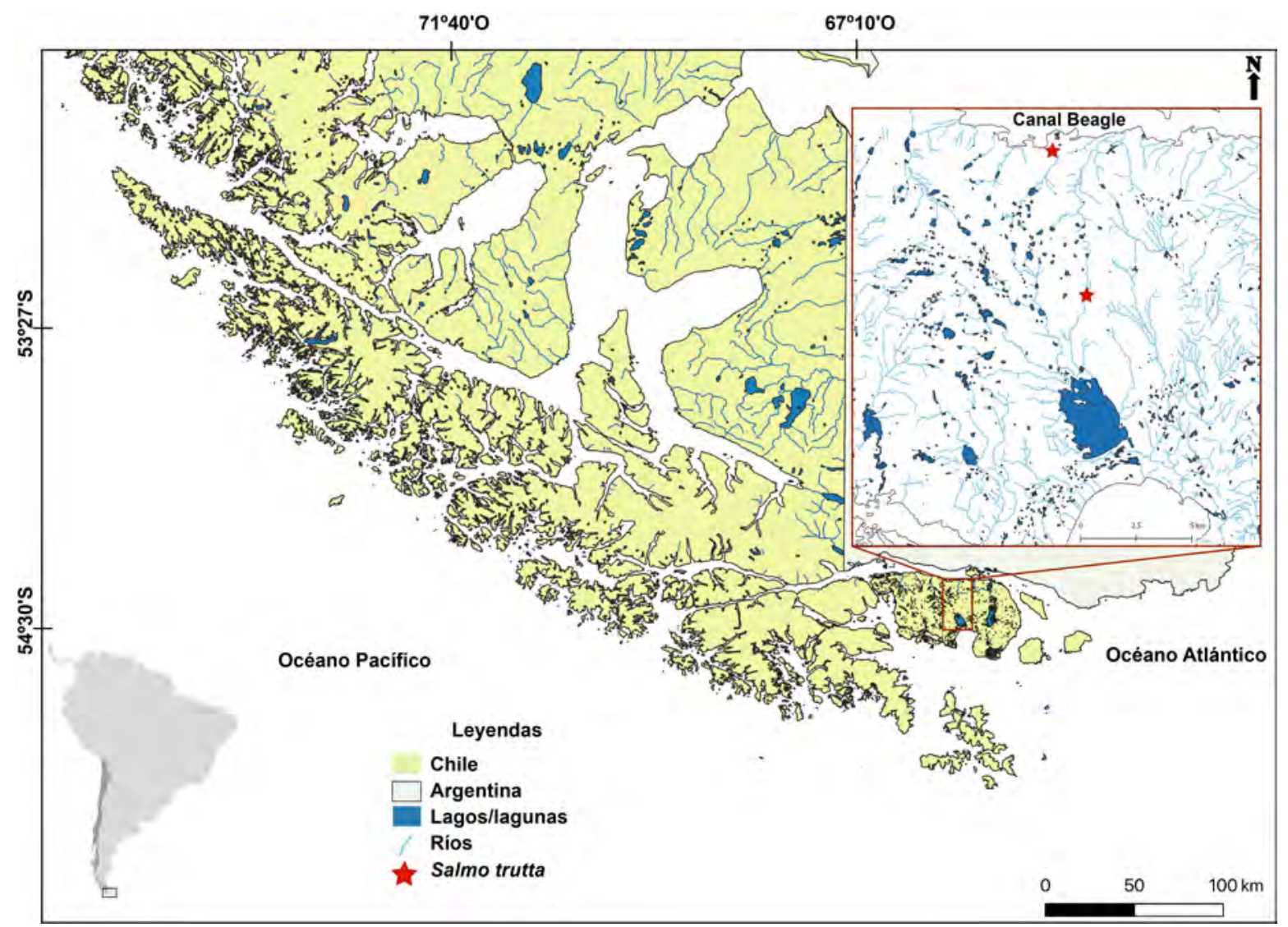

Fig. 1. Ubicación de isla Navarino con sus principales cuencas hidrográficas, al sur de Sudamérica.

Zoom en los sitios que se detectó la presencia de S. trutta.

(2006), Moorman et al. (2009) y MaldonadoMárquez et al. (2020). Entre los dos primeros estudios se produce una dicotomía sobre si realmente existe o no la presencia de $S$. trutta en isla Navarino, puesto que Anderson et al. (2006) reportan dicha especie para Navarino y Moorman et al. (2009) no registran la presencia de trucha marrón, siendo que abarcó un área de $16,397 \mathrm{~m}^{2}$ de cuerpos de agua dulce estudiados. Moorman et al. (2009) argumentan que el reporte de Anderson et al. (2006) se debe a una identificación errónea de los ejemplares de S. fontinalis. Por este motivo, el objetivo de esta nota es entregar nueva evidencia sobre la presencia de $S$. trutta en isla Navarino.

La obtención de muestras se enmarca dentro del Núcleo Milenio de Salmónidos Invasores INVASAL. La toma de muestras y reporte de $S$. trutta (Fig. 1) se basó en dos metodologías: a) uso de mochila de electro pesca
(Halltech, HT2000B/MK6, Guelph, Ontario, Canadá) y b) pesca tradicional (caña de pescar y señuelos). Se realizaron dos campañas de muestreo en las cuencas del río Windhond $\left(55^{\circ} 01,722^{\prime} \mathrm{S}, \quad 67^{\circ} 35,873^{\prime} \mathrm{O}\right)$ y río Róbalo (5456,494'S, 67³9,246'O). El río Windhond fue muestreado durante febrero del año 2019 y se recorrieron 5 tramos de 85 metros cada uno. En el río Róbalo se recorrieron 2 tramos de 85 metros en junio del año 2019. En cada tramo de los ríos, se efectuaron pasadas simples durante 1200 segundos de pesca eléctrica. Durante febrero de 2020 en el río Windhond $\left(55^{\circ} 03,983^{\prime} \mathrm{S}, 67^{\circ} 36,487^{\prime} \mathrm{O}\right)$, se realiza una nueva expedición en la que se utilizan artes de pesca tradicional (caña de pescar con señuelos) y se recorrió un total de $14 \mathrm{~km}$ durante 5 días en dirección sur hasta llegar al lago Windhond, dentro de los cuales se pesca efectivamente en 6 $\mathrm{km}$ durante la totalidad de los días, debido a la 


\section{A) Salmo trutta en río Róbalo}

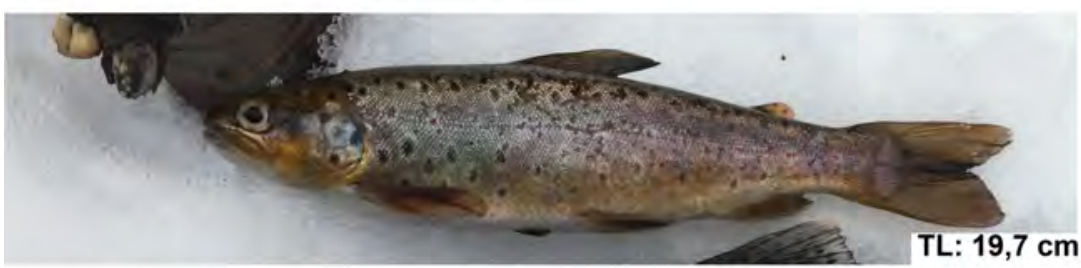

B) Salmo trutta en río Windhond

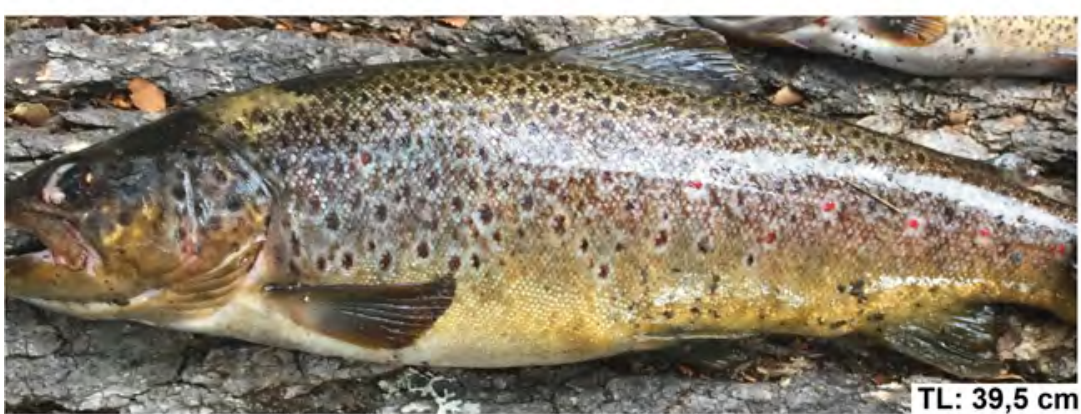

Fig. 2. Individuos capturados de S. trutta en las dos cuencas estudiadas.

A) río Róbalo, capturado el 2019 y B) río Windhond, capturado el 2020.

Longitud total (TL) es registrada para cada individuo en el borde inferior derecho de cada fotografía.

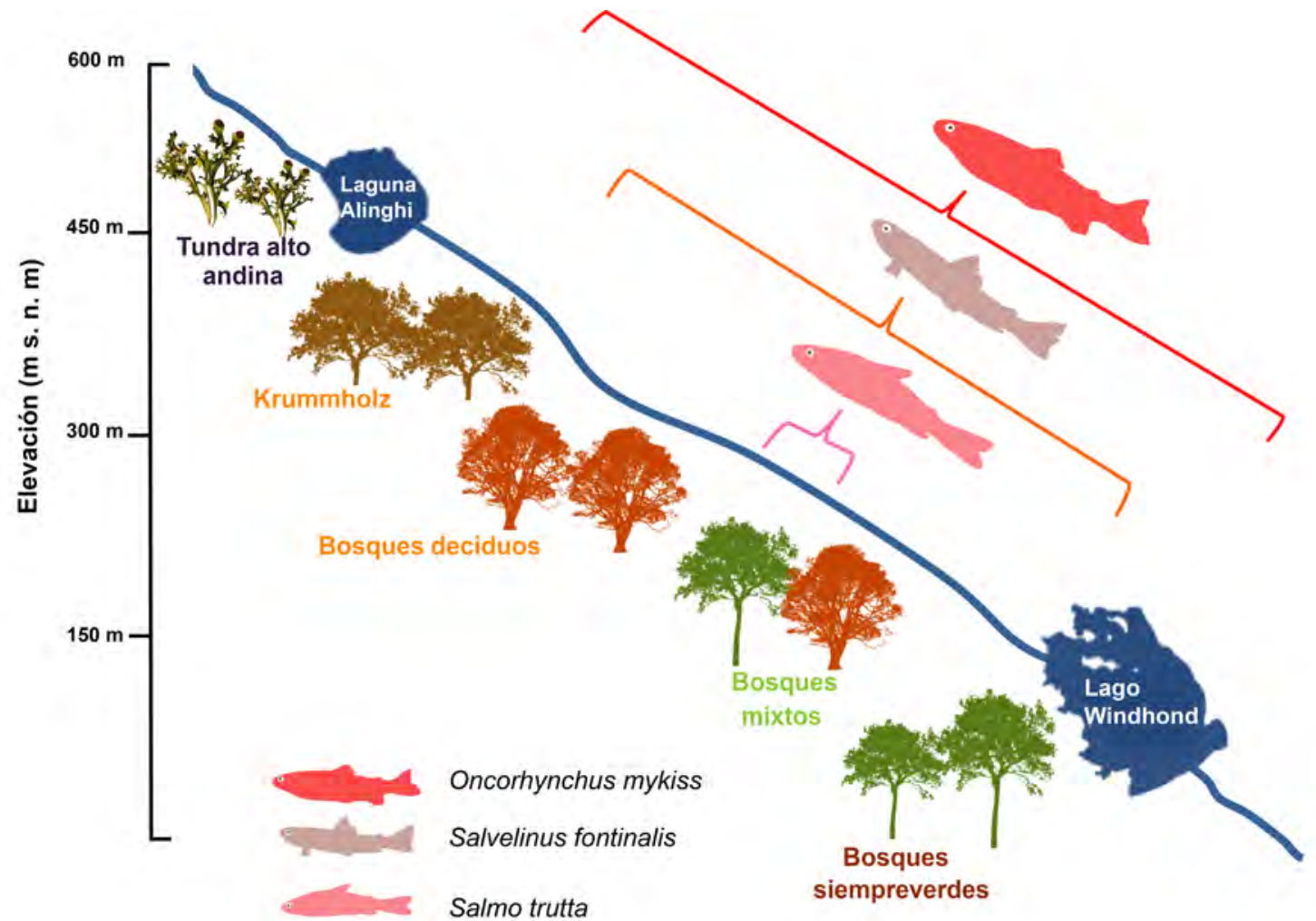

Fig. 3 Composición de ictiofauna (2019-2020) del río Windhond a lo largo de un gradiente altitudinal y vegetacional. Inicio desde laguna Alinghi y termino del gradiente en lago Windhond. El largo de cada barra indica la presencia de la especie en los diferentes tramos del río; la iconografía es especie-especifica. 
dificultad de acceso a la cuenca. Los especímenes capturados de $S$. trutta fueron eutanasiados con aceite de clavo de olor, siguiendo los procedimientos descritos por Fernandes et al. (2017); además fueron medidos con una regla estándar de $50 \mathrm{~cm}$ y pesados en una balanza OHAUS ${ }^{\circledR}$ (Parsippany, NJ, USA). Los individuos fueron identificados siguiendo la metodología descrita por McPhail y Carveth (1993).

Durante la campaña de 2019 en el río Windhond no se reportó la presencia de $S$. trutta en el sitio de estudio, sino sólo la presencia de la especie O. mykiss. En el río Róbalo para 2019 se registra la presencia de O. mykiss, S. fontinalis, O. kisutch (Walbaum, 1792) y S. trutta (Maldonado-Márquez et al. 2020). Este último individuo capturado corresponde a una hembra inmadura de un peso total de $69 \mathrm{~g}$ y longitud total de 19,7 cm. Estos hallazgos concuerdan con Maldonado-Márquez et al. (2020) para la riqueza de especies no nativas en esta cuenca. En febrero de 2020 se reporta la presencia de $O$. mykiss, $S$. fontinalis y se logra una captura de S. trutta (Fig. 2) en el río Windhond. Este último individuo capturado corresponde a una hembra sexualmente madura de un peso total de $1,98 \mathrm{~kg}$ y longitud total de $39,5 \mathrm{~cm}$.

El río Windhond es particularmente interesante debido a que tiende a presentar un ordenamiento espacial en la distribución de las especies (Fig. 3). La única especie que tiene distribución completa a lo largo de la cuenca es $O$. mykiss y a medida que se disminuye altitudinalmente sus abundancias van decreciendo. Esto es contrario al caso de $S$. fontinalis que no se encuentra distribuida en toda la cuenca, pero sus abundancias van en aumento a medida que se decrece en la altitud llegando al lago Windhond. Cabe destacar que $S$. trutta se encuentra relegada sólo a un tramo del río y se capturó únicamente un ejemplar cercano a una castorera, en la cual el caudal del río disminuye en comparación con los otros tramos muestreados.

En general, se acepta que $S$. trutta posee una amplia distribución a nivel mundial y Chile no es excepción, debido a su introducción a inicios del siglo XX (Basulto, 2003) y para Magallanes en 1927 (Traba \& Ríos, 1985). La literatura científica sobre esta especie en isla Navarino presenta una dicotomía sobre su presencia, sugiriendo que el canal Beagle representa una barrera biogeográfica para el establecimiento de S. trutta a los cuerpos interiores de agua dulce, esto debido a las condiciones ambientales que presenta el canal (Moorman et al. 2009). Planteamos que el canal Beagle no presenta una barrera biogeográfica para los salmónidos, puesto que recientemente se estableció una población de O. kisutch en la cuenca del río Róbalo (Maldonado-Márquez et al. 2020).

En sumatoria al reciente reporte de Maldonado-Márquez et al. (2020), y los registros de esta nota, se evidencia la presencia de $S$. trutta en isla Navarino, habitando dos cuerpos de agua diferentes. Aunque sus abundancias son relativamente bajas en comparación con otros salmónidos presentes en la isla, estas no deben ser entendida como una señal de despreocupación, debido a las implicancias negativas que pueden presentarse en el ecosistema, como el cambio en la estructura trófica y pérdida de biodiversidad nativa. Tanto O. mykiss y S. trutta son especies altamente dominantes sobre la fauna nativa y representan sobre el $80 \%$ de la biomasa total en la mayoría de las corrientes de tercer a cuarto orden del sur de Chile (Soto et al. 2006). Esto es particularmente alarmante, considerando que ambas especies están catalogadas dentro de las 100 especies invasoras más dañinas del mundo (Lowe et al. 2000; Courchamp, 2013) y actualmente reportadas para las aguas continentales de la $\mathrm{RBCH}$, siendo la riqueza de especies no nativa mayor (cuatro) a las nativas (tres) (Moorman et al. 2009; MaldonadoMárquez et al. 2020).

\section{AGRADECIMIENTOS}

Este trabajo es parte de las líneas de investigación del Núcleo Milenio de Salmónidos Invasores (INVASAL). Los autores agradecen especialmente a Javier Estay por el apoyo logístico en terreno y la fotografía de $S$. trutta en el río Windhond. También se agradece a Sergio González por la edición de figuras y al Instituto de Ecología y Biodiversidad a través de Fondo Basal ANID-AFB170008. Finalmente se agradece a los evaluadores anónimos por sus valiosas contribuciones y comentarios al manuscrito. 


\section{DECLARACIÓN DE ÉTICA}

La captura de los ejemplares empleados para este estudio se realizó conforme a: Resoluciones Exentas No3443-2017 y N4231-2017, ambas de 2017 de Subsecretaría de Pesca y Acuicultura; Certificado número 043/A/2019 del Subcomité de Bioseguridad del Comité de Ética Científico de la Universidad de Magallanes; Ley N²0.256 y sus modificaciones que Establece Normas sobre Pesca Recreativa; y, Decreto Supremo (MINECON) N¹03 de 2012 que Establece Aparejos Propios de Pesca para efectos de Pesca Recreativa y Submarina.

\section{LITERATURA CITADA}

Anderson, C., Rozzi, R., Torres-Mura, J., Mcgehee, S., Sherriffs, M., Schüttler, E., \& Rosemond, A. (2006). Exotic vertebrate fauna in the remote and pristine sub-Antarctic Cape Horn Archipelago, Chile. Biodiversity and Conservation, 15, 3295-3313.

Basulto, S. (2003). El largo viaje de los salmones: una crónica olvidada, propagación y cultivo de especies acuáticas en Chile. Editorial Maval.

Campos, H. (1970). Introducción de especies exóticas y su relación con los peces de agua dulce de Chile. Noticiario Mensual del Museo Nacional de Historia Natural, 14, 3-9.

Courchamp, F. (2013). Monster fern makes IUCN invader list. Nature 498, 37.

Fernandes, I. M., Bastos, Y. F., Barreto, D. S., Lourenco, L. S., \& Penha, J. M. (2017). The efficacy of clove oil as an anaesthetic and in euthanasia procedure for smallsized tropical fishes. Brazilian Journal of Biology, 77, 444-450.

Lowe, S., Browne, M., \& Boudjelas, S. (2000). 100 of the world's worst invasive alien species. A selection from the global invasive species database. Invasive Species
Specialist Group. Hollands Printing Ltd.

Maldonado-Márquez, A., Contador, T., Rendoll-

Cárcamo, J., Moore, S., Pérez-Troncoso,

C., Gómez-Uchida, D., \& Harrod, C. (2020). Southernmost distribution limit for endangered Peladillas (Aplochiton taeniatus) and non-native coho salmon (Oncorhynchus kisutch) coexisting within the Cape Horn biosphere reserve, Chile. Journal of Fish Biology, 1-6.

McPhail, J. D., \& Carveth, R. (1993). Field key to the freshwater fishes of British Columbia. British Columbia Ministry of Environment, Fisheries Branch, Resource Inventory Committee. Victoria, British Columbia: Queen's Printer.

Moorman, M., Eggleston, D., Anderson, C., Mansilla, A., \& Szejner, P. (2009). Implications of Beaver Castor canadensis and Trout Introductions on Native Fish in the Cape Horn Biosphere Reserve, Chile. Transactions of the American Fisheries Society, 138, 306-313.

Rozzi, R., Massardo, F., Anderson, C. B., Heidinger, K., \& Silander, J. A. (2006). Ten principles for biocultural conservation at the southern tip of the Americas: the approach of the Omora Ethnobotanical Park. Ecology and Society, 11, 43-70.

Soto, D., Arismendi, I., González, J., Guzmán, E., Sanzana, J., Jara, F., ... Lara, A. (2006). Southern Chile, trout and salmon country: invasion patterns and threats for native species. Revista Chilena de Historia Natural, 79, 97-117.

Traba, R., \& Ríos, C. (1985). Nota sobre la dieta de Salmo trutta (L) en Tierra del Fuego, Magallanes. Anales del Instituto de la Patagonia, 16, 87-90.

Vila, I., Fuentes, L., \& Saavedra, M. (1999). Ictiofauna en los sistemas límnicos de la Isla Grande, Tierra del Fuego, Chile. Revista Chilena de Historia Natural, 72, 273-284. 